\title{
Load Flow Analysis After the Entry of Renewable Power Plants in the Sulselrabar System
}

\author{
Muhammad Ruswandi Djalal $^{* 1}$, Makmur Saini ${ }^{1}$, A.M. Shiddiq Yunus ${ }^{2}$ \\ ${ }^{1}$ Department of Mechanical Engineering, Energy Generation Study Program, State Polytechnic of Ujung Pandang \\ J1. Perintis Kemerdekaan KM.10, Makassar, Indonesia \\ ${ }^{2}$ Department of Mechanical Engineering, Energy Conversion Study Program, State Polytechnic of Ujung Pandang \\ Jl. Perintis Kemerdekaan KM.10, Makassar, Indonesia \\ *Corresponding author, e-mail: wandi@poliupg.ac.id
}

\begin{abstract}
Power flow analysis in an electric power system is an analysis that reveals the performance of an electric power system and the flow of power (active and reactive) for certain conditions when the system is working. The analysis was carried out using the ETAP 16.00 software, the method used was the newton rapshon by taking a case study of normal conditions. From the results of the study, it can be seen that the power flow that occurs in each channel of the $150 \mathrm{kV}$ system in the South Sulawesi system. The amount of active power $(M W)$ that occurs during normal conditions based on the simulation is $1730.87 \mathrm{MW}$, where the active power is the largest, which is 171 MW from BUS15_TLASA to BUS13_SGMNSA. For the voltage data, there is a slight comparison of the voltage during the simulation compared to the PLN data.
\end{abstract}

Keywords: Power flow, active power, voltage, simulation, software ETAP 16.

\section{Introduction}

In the 1970s Renewable energy was first recognized as a way to offset energy developments with fossil fuels and nuclear. Indonesia's potential for renewable energy for electricity has reached 443 GW, including wind, geothermal, water and micromini hydro, solar bioenergy, and ocean waves. One of the renewable energies that the government is now ogling about is wind power or more commonly called wind power. Several eastern regions in Indonesia have the potential to generate electricity due to their wind speed. The wind speeds that have the potential to generate electricity are Oelbuluk, NTT the average speed is $6.1 \mathrm{~m} / \mathrm{s}$, Sidrap, South Sulawesi the average speed is $6.43 \mathrm{~m} / \mathrm{s}$ and Jeneponto, South Sulawesi the average is $7.96 \mathrm{~m} / \mathrm{s}$.

South Sulawesi itself, which is an industrial area in Eastern Indonesia, often experiences blackouts due to a power deficit. The blackout that occurs certainly affects the production process of industrial companies. Industries that require large electricity consumption, such as the food processing and plastic industries, sometimes have to experience obstacles due to long hours of blackouts. In addition, the number of electricity consumers continues to grow. From the results of the estimated number of customers, it is known that the amount of power needed or connected power in the following years, the amount of connected power in the household sector in 2015 is $1,296467,311 \mathrm{VA}$ and it is estimated that in 2025 it will increase to $2,597,148,624 \mathrm{VA}$. Therefore, the government is encouraged to build wind power plant installations in the South Sulawesi area which will later affect the distribution of electricity in the Sulserabar system.

After the presence of the Wind Power Plant (WPP), it can increase the stock of available power capacity in the South, Southeast, and West Sulawesi (Sulseltrabar) regions, thus increasing the number of burdens that must be borne. For this reason, it is necessary to conduct a power flow analysis to determine the overall condition of the electric power system in the current Sulselrabar system. Power flow analysis in an electric power system is an analysis that reveals the performance of an electric power system and the flow of power (real and reactive) for certain conditions when the system is working [1]. The main result of the power flow is the magnitude and phase angle of the voltage on each line (bus), the real power and reactive power present in each line 


\section{[2].}

In this study, an analysis of the system under normal conditions was carried out. Under normal conditions it will be known how much active power and reactive power occur and the Performance Index (IP) obtained from the calculation. The calculation of the power flow for the electric power system in the Sulselrabar System section after the entry of renewable plants if done manually will be very complicated and requires a lot of time, therefore in this study computer software was used to simplify and speed up the process of calculating the power flow. ETAP (Electrical Transient Analysis Program) Power Station is a software that can be used for calculating the flow of power in an electric power system. By using the ETAP Power Station 16.00 software, you will be able to analyze a very wide electric power system and many conditions [3].

Previously, there have been studies discussing power flow analysis, both the GaussSeidel method to the Newton Raphson method, as well as under normal and contingency conditions, among others [4] which discusses power flow analysis in the Sulselrabar system when renewable power has not yet entered, [5] discusses the analysis of the power flow of the electric power system in the texturizing section at PT Asia Pacific Fibers Tbk Kendal, [6] discusses the voltage analysis of each bus in the Gorontalo electric power system through power flow simulation, [7] discusses the study of power flow in the South Sulawesi electrical system, [8] discusses the contingency analysis of the Riau electric power system using the Newton Raphson power flow method, [9] discusses the load flow analysis on the East Kalimantan interconnection system. From this research, the development of power flow analysis needs to be carried out such as an up to date analysis by testing several case studies. The current Sulselrabar system continues to develop [10], especially after the inclusion of several renewable plants. The characteristics of the power flow need to be reviewed to see the characteristics of the system. In this study, a power flow analysis approach will be proposed for the entry of renewable power plants in the Sulselrabar system.

\section{Research Method}

The research was conducted by simulating the electric power system of the Sulserabar system of PT. PLN AP2B Sulselrabar Region due to the entry of renewable power plants using the ETAP 16.00 application. The modeling of the system to be carried out, adjusts the parameters needed and has been accepted by the researcher when collecting data. The simulated system is designed in such a way as to achieve similarity to the real $150 \mathrm{kV}$ Sulserabar network system.

The initial step of the research is to determine the parameters or technical data supporting the desired value. This data will be obtained when researchers carry out data collection at PT. PLN AP2B for Sulselrabar Region, Makassar. After the data and method analysis have been carried out, the next step is to design a single line diagram of a $150 \mathrm{kV}$ network for the Sulselrabar system on the ETAP 16.00 application which is useful for facilitating the next stage.

Network modeling is the next step by entering data in each installed component with data that is already available, the modeling stage plays an important role for this research because the network is made according to the real conditions of the Sulserabar system. After the design and modeling is complete, the next step is to run load flow on the ETAP 16.00 application to ensure the modeling runs well. After successfully simulating, the results of the power flow are obtained, then identify the parameters of active power and bus voltage under normal conditions.

The next step, the researchers began to simulate contingency by removing one channel installed in the selected system, and conducting a power flow analysis during contingency. Then analyze the power flow generated during contingency by identifying the parameters needed for the calculation of the Performance Index. The active power and bus voltage will be recorded at the time of line disconnection.

\section{Results and Discussions}

The currently active Sulselrabar electricity system consists of 21 generating units, namely 6 PLTA, 8 PLTU, 1 PLTG, 1 PLTGU, 3 PLTMH and 2 WPP. operating at $150 \mathrm{kV}$. The Sulselrabar Electricity System is dominated by Steam Power Plants (PLTU) such as PLTU Sengkang, PLTU Barru, PLTU Jeneponto and PLTU Mamuju, each of which has a different generating capacity. The Sulselrabar bus system numbering is shown in Table 1.

\section{1. Power Flow Simulation Results}

Analysis using ETAP 16.00 Software was carried out on the Sulselrabar System with normal loading and generation conditions using operating data on 
Tuesday, May 21 2019, at 15.00 WITA. As a comparison data, the results of the analysis will be compared with the results of the power flow analysis study from the research [3] as data before the entry of WPP, the results obtained are as follows

The simulation with the ETAP 16.00 software uses the Newton Raphson Method which is completed in the 2nd iteration, resulting in data on Active Power, Reactive Power, Current and Power Factor Efficiency flowing in each channel based on the simulation results of power flow when the Sulselrabar system under normal conditions is given in Table 2.

On the results of the power flow from the 43 Bus interconnection system $150 \mathrm{kV}$ Sulselrabar System, it can produce the following data conclusions,

a) The total active power contained in the channel under normal conditions is 1730.87 MW, where the active power is the largest, which is $171 \mathrm{MW}$ from BUS15_TLASA to BUS13_SGMNSA.

b) The largest reactive power is 25.68 Mvar from BUS8_PANKEP to BUS8_BOSOWA.

c) The highest power factor efficiency reached $100 \%$ occurred from BUS21_SENGKANG to BUS16_SIDRAP, while the lowest efficiency occurred on the WPP Sidrap Bus channel to BUS16_SIDRAP which was $19.72 \%$.

TABLE I

BUS NUMBER

\begin{tabular}{cccc}
\hline \hline ID BUS & $\mathrm{kV}$ & ID BUS & $\mathrm{kV}$ \\
\hline BU28_MAROS & 150 & BUS21_SENGKANG & 150 \\
BUS1_BAKARU & 150 & BUS22_BONE & 150 \\
BUS2_POLMAS & 150 & BUS23_SINJAI & 150 \\
BUS3_MAJENE & 150 & BUS24_BLKMBA & 150 \\
BUS4_PINRANG & 150 & BUS25_JNPNTO & 150 \\
BUS5_PARE & 150 & BUS26_PLTUMmuju & 150 \\
BUS6_SUPPA & 150 & BUS27_PUNAGAYA & 150 \\
BUS7_BARRU & 150 & BUS28_ENRKG & 150 \\
BUS8_PNGKEP & 150 & BUS29_WPP Sidrap & 150 \\
BUS9_BOSOWA & 150 & BUSBNTAENG & 150 \\
BUS10_TELLO & 150 & BusBOLANGI & 150 \\
BUS11_TLAMA & 150 & BUS26_PANGKEP70 & 70 \\
BUS12_PKANG & 150 & BUS27_TNASA70 & 70 \\
BUS13_SGMNS & 150 & BUS29_MNDAI & 70 \\
BUS14_TBNGA & 150 & BUS30_DAYA & 70 \\
BUS15_TLASA & 150 & Bus31_TELLO70 & 70 \\
BUS16_SIDRAP & 150 & BUS32_BRLOE & 70 \\
BUS17_MKALE & 150 & Bus33_TLAMA70 & 70 \\
BUS18_PALOPO & 150 & BUS34_BNTLA & 70 \\
BUS19_MMUJU & 150 & Bus35_TELLO30A & 30 \\
BUS20_SPPENG & 150 & & \\
\hline \hline
\end{tabular}

TABLE II

Results Of Power Flow Simulation Of Each Bus In INTERCONNECTION SYSTEM 150 KV SULSELRABAR

\begin{tabular}{|c|c|c|c|c|}
\hline \multirow[b]{2}{*}{ ID } & \multicolumn{2}{|c|}{ Lines } & \multicolumn{2}{|c|}{ Load Flow } \\
\hline & From & To & $\begin{array}{c}\mathrm{P} \\
(\mathrm{MW})\end{array}$ & $\begin{array}{c}\text { Q } \\
\text { (Mvar) }\end{array}$ \\
\hline \multirow[t]{2}{*}{1} & BTLA 150 & BD_TELLO2 & 36,04 & 11,29 \\
\hline & & BusBOLANGI & 8,64 & $-1,33$ \\
\hline \multirow[t]{2}{*}{2} & BU28 & BUS13 & 0,83 & $-2,63$ \\
\hline & & BD_MROS & 16,94 & 6,19 \\
\hline \multirow[t]{2}{*}{3} & BUS1 & BUS'2 & 56,59 & 12,01 \\
\hline & & BUS1 & 57,48 & $-9,52$ \\
\hline 4 & BUS2 & BD_PLMAS & 4,75 & 1,68 \\
\hline 5 & BUS3 & BUS2 & 4,07 & 2,86 \\
\hline \multirow[t]{2}{*}{6} & BUS4 & BUS1 & 71,08 & $-18,71$ \\
\hline & & BUS8 & 7,40 & $-2,68$ \\
\hline \multirow[t]{2}{*}{7} & BUS5 & BUS4 & 95,73 & $-7,35$ \\
\hline & & BUS2 & 55,24 & $-12,57$ \\
\hline 8 & BUS6 & BUS5 & 19,98 & 5,83 \\
\hline \multirow[t]{2}{*}{9} & BUS7 & BUS8 & 27,27 & $-4,16$ \\
\hline & & BUS5 & 13,31 & $-1,34$ \\
\hline 10 & BUS8 & BUS9 & 13,97 & 25,68 \\
\hline 11 & BUS9 & BUS8 & 13,88 & $-25,79$ \\
\hline \multirow[t]{4}{*}{12} & BUS10 & BUS9 & 31,34 & $-8,58$ \\
\hline & & BUS8 & 16,06 & $-18,28$ \\
\hline & & BUS12 & 32,66 & 9,69 \\
\hline & & BUS11 & 42,62 & 8,00 \\
\hline \multirow[t]{2}{*}{13} & BUS11 & BONTOALA150 & 36,10 & $\mathrm{q} 11,19$ \\
\hline & & Bus33 & 2,99 & 0,10 \\
\hline \multirow[t]{2}{*}{14} & BUS12 & BUS10 & 32,61 & $-9,81$ \\
\hline & & BusBOLANGI & 7,82 & $-0,50$ \\
\hline \multirow[t]{2}{*}{15} & BUS13 & BUS14 & 24,90 & 4,42 \\
\hline & & BUS10 & 124,29 & 8,68 \\
\hline \multirow[t]{2}{*}{16} & BUS14 & BUS13 & 24,88 & $-4,51$ \\
\hline & & BUS_PNAGAYA & 131,80 & $-24,49$ \\
\hline \multirow[t]{2}{*}{17} & BUS15 & BUS13 & 171,25 & 23,11 \\
\hline & & BUS5 & 61,80 & $-13,31$ \\
\hline \multirow[t]{3}{*}{18} & BUS16 & ENRKG & 13,16 & $-0,02$ \\
\hline & & BUS17 & 20,21 & 3,38 \\
\hline & & BUS18_PALOPO & 15,06 & 4,60 \\
\hline 19 & BUS17 & ENRKG & 7,07 & $-4,32$ \\
\hline 20 & BUS19 & BUS3 & 9,33 & 3,31 \\
\hline 21 & BUS20 & BUS16 & 26,39 & 4,08 \\
\hline \multirow[t]{2}{*}{22} & BUS21 & BUS16 & 59,13 & $-0,32$ \\
\hline & & BUS20 & 17,31 & $-12,77$ \\
\hline 23 & BUS22 & BUS20 & 5,69 & 12,93 \\
\hline 24 & BUS23 & BUS22_BONE & 9,36 & $-2,95$ \\
\hline \multirow[t]{2}{*}{25} & BUS24 & BUS22_BONE & 14,76 & $-1,36$ \\
\hline & & BUS23_SINJAI & 21,68 & 1,72 \\
\hline \multirow[t]{3}{*}{26} & BUS25 & BUSBNTTAENG & 16,12 & $-9,82$ \\
\hline & & BUS24_BLKBA & 26,11 & $-20,06$ \\
\hline & & BD_JNPNTO & 12,80 & 4,70 \\
\hline 27 & $\begin{array}{l}\text { BusPLTUMa } \\
\text { muju }\end{array}$ & $\begin{array}{l}\text { BUS19_MAMUJ } \\
\text { U }\end{array}$ & 20,31 & 5,95 \\
\hline \multirow[t]{2}{*}{28} & BUS_PUNAG & BUS25_JNPNTO & 27,55 & $-12,87$ \\
\hline & AYA & BUS15_TLASA & 132,64 & 29,62 \\
\hline 29 & ENRKG & BUS17_MKALE & 7,09 & 1,97 \\
\hline 30 & WPP Sidrap & BU28_MAROS & 13,21 & 0,29 \\
\hline & & BUS16_SIDRAP & 2,25 & $-11,20$ \\
\hline
\end{tabular}

If the analysis is compared as data after the entry of the WPP with the analysis before the entry of the WPP using data from, then what happens is that the Active Power $(\mathrm{P})$ that occurs in each channel has increased. The channel that experienced the highest increase in power was BUS7_BARRU to BUS8_PANKEP with an increase in active power (P) of $66.62 \mathrm{MW}$. This can be seen in Table 3 . 
TABLE III

Results Of Power Flow Simulation Of The $150 \mathrm{Kv}$ INTERCONNECTION SYSTEM IN SULSELRABAR BEFORE AND AFTER Wind POWER INSTALLING

\begin{tabular}{|c|c|c|c|c|c|}
\hline \multirow{2}{*}{\multicolumn{2}{|c|}{ LINES }} & \multicolumn{4}{|c|}{ LOAD FLOW } \\
\hline & & \multicolumn{2}{|c|}{ BEFORE } & \multicolumn{2}{|c|}{ AFTER } \\
\hline From & To & $\begin{array}{c}\mathrm{P} \\
(\mathrm{MW})\end{array}$ & $\begin{array}{c}\mathrm{Q} \\
\text { (Mvar) }\end{array}$ & $\begin{array}{c}\mathrm{P} \\
(\mathrm{MW})\end{array}$ & $\begin{array}{c}\mathrm{Q} \\
\text { (Mvar) }\end{array}$ \\
\hline BU28 & BUS13 & 37,36 & 1,53 & 42,83 & $-2,63$ \\
\hline BUS1 & BUS2 & 11,8 & $-0,11$ & 56,59 & 12,01 \\
\hline BUS3 & BUS19 & 3,93 & 0,52 & 4,07 & 2,86 \\
\hline BUS4 & BUS5 & 6,99 & 0,95 & 71,08 & $-18,71$ \\
\hline BUS5 & BUS8 & 42,76 & $-0,5$ & 7,40 & $-2,68$ \\
\hline BUS6 & BUS5 & 28,46 & 4,3 & 19,98 & 5,83 \\
\hline BUS7 & BUS8 & 62,46 & $-2,79$ & 27,27 & $-4,16$ \\
\hline BUS8 & BUS9 & 27,59 & 4,17 & 13,97 & 25,68 \\
\hline BUS9 & BUS10 & 6,91 & $-7,26$ & 13,88 & $-25,79$ \\
\hline \multirow{2}{*}{ BUS10 } & BUS12 & 14,93 & $-4,92$ & 32,66 & 9,69 \\
\hline & BUS11 & 9,2 & $-1,72$ & 42,62 & 8,00 \\
\hline BUS11 & Bus33 & 10,44 & $-5,14$ & 2,99 & 0,10 \\
\hline \multirow[t]{2}{*}{ BUS13 } & BUS14 & 13,53 & 6,96 & 24,90 & 4,42 \\
\hline & BUS10 & 40,07 & 7,35 & 124,29 & 8,68 \\
\hline BUS15 & BUS13 & 12,4 & 9,32 & 171,25 & 23,11 \\
\hline \multirow{2}{*}{ BUS16 } & BUS5 & 5,6 & $-4,39$ & 61,80 & $-13,31$ \\
\hline & BUS17 & 8,41 & 1,27 & 20,21 & 3,38 \\
\hline BUS17 & BUS18 & 8,23 & 3,11 & 15,06 & 4,60 \\
\hline BUS20 & BUS16 & 10,53 & 3,87 & 26,39 & 4,08 \\
\hline \multirow{2}{*}{ BUS21 } & BUS16 & 49,8 & $-1,8$ & 59,13 & $-0,32$ \\
\hline & BUS20 & 33,65 & $-10,41$ & 17,31 & $-12,77$ \\
\hline
\end{tabular}

The number of losses obtained is $12,123 \mathrm{~kW}$, where the largest losses are in the BAKARUPINRANG line, namely $1,662 \mathrm{~kW}$, in addition to transmission losses there are also losses in the transformer. For more details, see the literature. The Table 4 shows the losses that occur in the transmission line.

\section{2. Active Power and Reactive Power on Bus Loading}

From the simulation results using ETAP with Newton Raphson method, it can be seen the difference in active power, reactive power and PF that occurs in each channel.

Bus Loading which has the largest active power is found in the BOSOWA Loading bus which is 231.5 MW with 45 Mvar reactive power and $98.16 \%$ Power Factor, while the complete results can be seen in Table 5.

Next, compare the results of the power flow analysis simulation on the bus loading before entering and after entering the WPP by taking comparative data from the study [3].

The total active power $(\mathrm{P})$ that occurs at the bus loading before the entry of WPP in the $150 \mathrm{kV}$ interconnection system is $455.61 \mathrm{MW}$, while the total active power $(\mathrm{P})$ after the entry of the WPP is 610.31 MW, more details can be seen in Table 6 .
TABLE IV

LOSSES IN TRANSMISSION LINE IN INTERCONNECTION SYSTEM $150 \mathrm{KV}$ SULSELRABAR

\begin{tabular}{|c|c|c|c|}
\hline \multirow{2}{*}{ NO } & \multirow{2}{*}{ LINES } & \multicolumn{2}{|c|}{ LOSSES } \\
\hline & & $\mathrm{kW}$ & kvar \\
\hline 1 & $\begin{array}{l}\text { TELLOLAMA- } \\
\text { BONTOALA4 }\end{array}$ & 64,0768 & $-103,1$ \\
\hline 2 & MAROS-BOLANGI & 20,4674 & $-3423,6$ \\
\hline 3 & $\begin{array}{l}\text { MAROS- } \\
\text { SUNGGUMINASA1 }\end{array}$ & 0,38356 & $-3577,1$ \\
\hline 4 & SIDRAP-MAROS1 & 10,8645 & $-820,25$ \\
\hline 5 & BAKARU-PINRANG & 1662,49 & 5059,31 \\
\hline 6 & BAKARU-POLMAS & 881,497 & 2489,23 \\
\hline 7 & POLMAS-MAJENE1 & 6,93801 & $-659,33$ \\
\hline 8 & POLMAS-PARE & 1152,06 & 2317,68 \\
\hline 9 & MAJENE-MAMUJU1 & 25,6558 & $-598,79$ \\
\hline 10 & PINRANG-PARE & 1261,56 & 3842,72 \\
\hline 11 & PARE-BARRU & 40,37 & $-998,27$ \\
\hline 12 & PARE-PANGKEP & 26,6489 & $-2183,2$ \\
\hline 13 & PARE-SIDRAP1 & 393,773 & 1186,23 \\
\hline 14 & SUPPA-PARE1 & 16,8107 & $-168,01$ \\
\hline 15 & BARRU-PANGKEP & 179,684 & $-498,15$ \\
\hline 16 & PANGKEP-BOSOWA & 93,3184 & $-114,66$ \\
\hline 17 & PANGKEP-TELLO & 137,747 & $-631,84$ \\
\hline 18 & BOSOWA-TELLO & 178,653 & $-25,277$ \\
\hline 19 & $\begin{array}{l}\text { TELLO- } \\
\text { PANAKUKKANG1 }\end{array}$ & 55,5004 & $-120,06$ \\
\hline 20 & $\begin{array}{l}\text { TELLO- } \\
\text { SUNGGUMINASA1 }\end{array}$ & 300,038 & 1831,26 \\
\hline 21 & TELLO-TELLOLAMA1 & 69,0172 & 25,4746 \\
\hline 22 & MAROS-SGMNSA & 17,1501 & $-3451,2$ \\
\hline 23 & $\begin{array}{l}\text { SUNGGUMINASA- } \\
\text { TNJUNGBUNGA1 }\end{array}$ & 22,7612 & $-86,716$ \\
\hline 24 & $\begin{array}{l}\text { TALLASA- } \\
\text { SUNGGUMINASA1 }\end{array}$ & 1413,88 & 9011,84 \\
\hline 25 & TALLASA-PUNAGAYA & 849,596 & 5122,88 \\
\hline 26 & SIDRAO-SENGKANG1 & 358,443 & 1237,18 \\
\hline 27 & SIDRAP-ENRKG & 53,9369 & $-2174,6$ \\
\hline 28 & SIDRAP-MAKALE1 & 132,788 & $-1679,9$ \\
\hline 29 & SIDRAP-SENGKANG2 & 358,443 & 1237,18 \\
\hline 30 & SIDRAP-SOPPENG1 & 194,753 & $-225,06$ \\
\hline 31 & MAKALE-PALOPO1 & 49,5934 & $-263,25$ \\
\hline 32 & SIDRAP-ENRKG2 & 18,931 & $-2349,8$ \\
\hline 33 & Linemauju & 113,131 & $-295,92$ \\
\hline 34 & SOPPENG-BONE1 & 45,6797 & $-783,58$ \\
\hline 35 & SOPPENG-SENGKANG1 & 46,2633 & $-651,54$ \\
\hline 36 & BONE-BULUKUMBA & 144,859 & $-1651,9$ \\
\hline 37 & BONE-SINJAI & 35,5433 & $-1069,1$ \\
\hline 38 & SINJAI-BULUKUMBA & 136,89 & $-473,88$ \\
\hline 39 & $\begin{array}{l}\text { BNTAENG- } \\
\text { BULUKUMBA4 }\end{array}$ & 46,9618 & $-557,06$ \\
\hline 40 & $\begin{array}{l}\text { JENEPONTO- } \\
\text { BULUKUMBA1 }\end{array}$ & 245,555 & 159,476 \\
\hline 41 & BNTAENG-JNPNTO & 80,1965 & $-430,04$ \\
\hline 42 & JENEPONTO-PNGYA & 29,278 & $-275,28$ \\
\hline 43 & PANGKEP70-MANDAI1 & 44,7319 & $-17,921$ \\
\hline 44 & $\begin{array}{l}\text { PANGKEP70- } \\
\text { TONASA701 }\end{array}$ & 21,0497 & $-11,209$ \\
\hline 45 & MANDAI-DAYA & 16,4574 & $-20,003$ \\
\hline 46 & MANDAI-TELLO70 & 56,8898 & 54,104 \\
\hline 47 & DAYA-TELLO70 & 144,823 & 215,283 \\
\hline 48 & TELLO70-BORONGLOE & 22,1431 & $-10,251$ \\
\hline 49 & $\begin{array}{l}\text { TELLOLAMA- } \\
\text { BONTOALA1 }\end{array}$ & 1,83994 & $-44,748$ \\
\hline 50 & TELLO30-BARAWAJA & 842,88 & 1192,8 \\
\hline
\end{tabular}


TABLE V

RESUlts OF POWER Flow Bus LOADING INTERCONNECTION SYSTEM 150KV SULSELRABAR

\begin{tabular}{|c|c|c|c|}
\hline NO & ID BUS & $\mathrm{P}(\mathrm{MW})$ & Q (Mvar) \\
\hline 1 & BD1_BNTLA & 5,97 & 0,00 \\
\hline 2 & BD1_BONE & 6,27 & 1,97 \\
\hline 3 & BD1_DAYA & 11,63 & 0,00 \\
\hline 4 & BD1_MNDAI & 7,98 & 2,56 \\
\hline 5 & BD1_PLPO & 15,47 & 0,30 \\
\hline 6 & BD1_PNGAYA & 0,74 & 0,11 \\
\hline 7 & BD1_PNKNG & 16,74 & 3,14 \\
\hline 8 & BD1_PNRNG & 24,38 & 6,34 \\
\hline 9 & BD1_TLAMA & 12,22 & 4,02 \\
\hline 10 & BD1_TLLASA & 16,04 & 4,58 \\
\hline 11 & BD2_BONE & 6,28 & 1,97 \\
\hline 12 & BD2_DAYA & 11,86 & 4,53 \\
\hline 13 & BD2_MNDAI & 11,02 & 0,00 \\
\hline 14 & BD2_PLPO & 14,52 & 6,16 \\
\hline 15 & BD2_PNKNG & 17,80 & 4,14 \\
\hline 16 & BD2_PNRNG & 1,00 & 0,01 \\
\hline 17 & BD2_TLAMA & 30,74 & 4,28 \\
\hline 18 & BD3_PNKNG & 30,59 & 7,69 \\
\hline 19 & BD_5 & 95,00 & 9,50 \\
\hline 20 & BD_9 & 89,80 & 15,00 \\
\hline 21 & BD_10 & 231,50 & 45,00 \\
\hline 22 & BD_BARRU & 4,53 & 1,35 \\
\hline 23 & BD_BKRU & 1,00 & 0,00 \\
\hline 24 & BD_BLKMBA & 0,74 & 1,61 \\
\hline 25 & BD_BNTAENG & 6,17 & 1,17 \\
\hline 26 & BD_BOLANGI & 16,41 & 3,79 \\
\hline 27 & BD_BOSOWA & 44,91 & 9,84 \\
\hline 28 & BD_BRLOE & 6,14 & 0,85 \\
\hline 29 & BD_BRWJA & 22,67 & 0,01 \\
\hline 30 & BD_ENRKG & 6,01 & 0,00 \\
\hline 31 & BD_JNPNTO & 12,79 & 3,63 \\
\hline 32 & BD_MJENE & 10,45 & 1,42 \\
\hline 33 & BD_MKLE & 2,97 & 0,13 \\
\hline 34 & BD_MMUJU & 21,72 & 2,83 \\
\hline 35 & BD_MROS & 16,93 & 4,82 \\
\hline 36 & BD_PARE & 17,63 & 4,07 \\
\hline 37 & BD_PLMAS & 4,75 & 1,52 \\
\hline 38 & BD_PNGKEP & 24,96 & 10,98 \\
\hline 39 & BD_SDRP & 17,40 & 5,68 \\
\hline 40 & BD_SGMNSA & 34,28 & 0,97 \\
\hline 41 & BD_SIWA & 6,25 & 1,41 \\
\hline 42 & BD_SNGKNG & 19,49 & 5,37 \\
\hline 43 & BD_SNJAI & 12,18 & 4,12 \\
\hline 44 & BD_SPPENG & 6,97 & 5,41 \\
\hline 45 & BD_TBNGA & 49,64 & 0,99 \\
\hline 46 & BD_TELLO & 35,31 & 14,13 \\
\hline 47 & BD_TELLO2 & 35,97 & 8,65 \\
\hline 48 & BD_TONASA & 2,33 & 21,83 \\
\hline
\end{tabular}

\section{3. Voltage Simulation Results on each Bus}

The voltage obtained from the simulation results of the power flow of each bus on the $150 \mathrm{kV}$ Sulselrabar interconnection system after the entry of the WPP can be seen in Table 7. So it can be concluded:

a) Bus $150 \mathrm{kV}$ the largest voltage before the entry of the WPP occurred at BUS22_BONE of $152.31 \mathrm{kV}$ or $101.54 \%$, while the largest voltage after the entry of the WPP occurred at BUS24_BLKMB of $152.31 \mathrm{kV}$ or $104.02 \%$.

b) The smallest voltage value before the entry of the WPP occurred at BUS9_BOSOWA with a value of $147.071 \mathrm{kV}$ or $98.05 \%$, while the voltage after the entry of the WPP occurred at BUS18_PALOPO with a voltage of $148.091 \mathrm{kV}$ or $98.72 \%$.

c) The same voltage value with PT. PLN data occurs in BUS1_BAKARU with a value of $150 \mathrm{kV}$ or $100 \%$. The following is a graphic image of the results of the $150 \mathrm{kV}$ analysis. This can be seen in Figure 1 and Table 7.

TABLE VI

Results Of Power Flow Simulation Of The $150 \mathrm{Kv}$ SULSELRABAR BUS LOADING INTERCONNECTION SYSTEM Before ANd AFTER Wind POWER ENTER

\begin{tabular}{|c|c|c|c|c|}
\hline \multirow{2}{*}{ BUS } & \multicolumn{2}{|c|}{ Before } & \multicolumn{2}{|c|}{ After } \\
\hline & $\mathrm{P}(\mathrm{MW})$ & Q (Mvar) & $\mathrm{P}(\mathrm{MW})$ & Q (Mvar) \\
\hline BD1_BNTLA & 7,99 & 0,00 & 5,97 & 0,00 \\
\hline BD1_BONE & 4,04 & 1,71 & 6,27 & 1,97 \\
\hline BD1_DAYA & 11,45 & 0,01 & 11,63 & 0,00 \\
\hline BD1_MNDAI & 7,86 & 1,52 & 7,98 & 2,56 \\
\hline BD1_PLPO & 12,78 & 4,91 & 15,47 & 0,30 \\
\hline BD1_PNKNG & 3,79 & 3,08 & 16,74 & 3,14 \\
\hline BD1_PNRNG & 12,51 & 2,14 & 24,38 & 6,34 \\
\hline BD1_TLAMA & 12,08 & 3,97 & 12,22 & 4,02 \\
\hline BD1_TLLASA & 0,68 & 0,10 & 16,04 & 4,58 \\
\hline $\mathrm{BD} 2 \mathrm{BONE}$ & 10,24 & 4,09 & 6,28 & 1,97 \\
\hline BD2_DAYA & 11,68 & 4,46 & 11,86 & 4,53 \\
\hline BD2_MNDAI & 10,86 & 0,01 & 11,02 & 0,00 \\
\hline BD2_PLPO & 8,72 & 1,74 & 14,52 & 6,16 \\
\hline BD2_PNKNG & 13,86 & 4,06 & 17,80 & 4,14 \\
\hline BD2_PNRNG & 7,23 & 4,68 & 1,00 & 0,01 \\
\hline BD2_TLAMA & 19,40 & 1,58 & 30,74 & 4,28 \\
\hline BD3_PNKNG & 26,44 & 7,54 & 30,59 & 7,69 \\
\hline BD_BARRU & 4,23 & 1,25 & 4,53 & 1,35 \\
\hline BD_BKRU & 2,29 & 0,19 & 1,00 & 0,00 \\
\hline BD_BLKMBA & 9,13 & 2,00 & 0,74 & 1,61 \\
\hline BD_BOSOWA & 20,56 & 9,96 & 44,91 & 9,84 \\
\hline BD_BRLOE & 7,09 & 9,96 & 6,14 & 0,85 \\
\hline BD_BRWJA & 5,23 & 0,00 & 22,67 & 0,01 \\
\hline BD_JNPNTO & 9,74 & 3,46 & 12,79 & 3,63 \\
\hline BD_MJENE & 5,21 & 1,73 & 10,45 & 1,42 \\
\hline BD_MKLE & 3,81 & 1,67 & 2,97 & 0,13 \\
\hline BD_MMUJU & 7,85 & 1,91 & 21,72 & 2,83 \\
\hline BD_MROS & 4,90 & 2,16 & 16,93 & 4,82 \\
\hline BD_PARE & 20,00 & 4,40 & 17,63 & 4,07 \\
\hline BD_PLMAS & 6,63 & 2,40 & 4,75 & 1,52 \\
\hline BD_PNGKEP & 13,38 & 6,56 & 24,96 & 10,98 \\
\hline BD_SDRP & 12,21 & 5,83 & 17,40 & 5,68 \\
\hline BD_SGMNSA & 11,90 & 4,30 & 34,28 & 0,97 \\
\hline BD_SNGKNG & 11,72 & 5,44 & 19,49 & 5,37 \\
\hline BD_SNJAI & 5,75 & 3,33 & 12,18 & 4,12 \\
\hline BD_SPPENG & 14,00 & 7,40 & 6,97 & 5,41 \\
\hline BD TBNGA & 27.00 & 11.24 & 49.64 & 0,99 \\
\hline BD_TELLO & 34,55 & 13,82 & 35,31 & 14,13 \\
\hline BD_TONASA & 36,64 & 21,33 & 2,33 & 21,83 \\
\hline
\end{tabular}




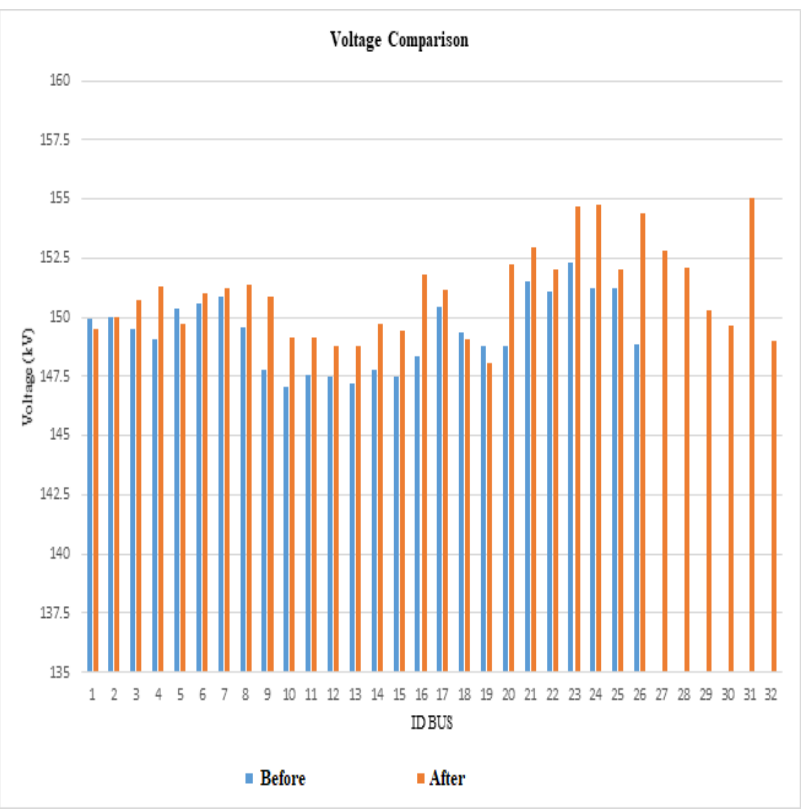

Fig. 1. 150kV voltage comparison graph from Power flow analysis and data from [3]

At Bus $70 \mathrm{kV}$, the largest voltage before the entry of the WPP occurred at Bus31_TELLO70 of 70.625 $\mathrm{kV}$ or $100.89 \%$, while the largest voltage after the entry of the WPP occurred at BUS32_BRLOE with a voltage value of $71.493 \mathrm{kV}$ or $102.13 \%$.

The smallest voltage value before the entry of the WPP occurs at BUS27_TNASA70 with a value of $69.951 \mathrm{kV}$ or $99.93 \%$, while the voltage after the entry of the WPP occurs at BUS34_BNTLA with a voltage value of $69.351 \mathrm{kV}$ or $99.07 \%$. This can be seen in Figure 2.

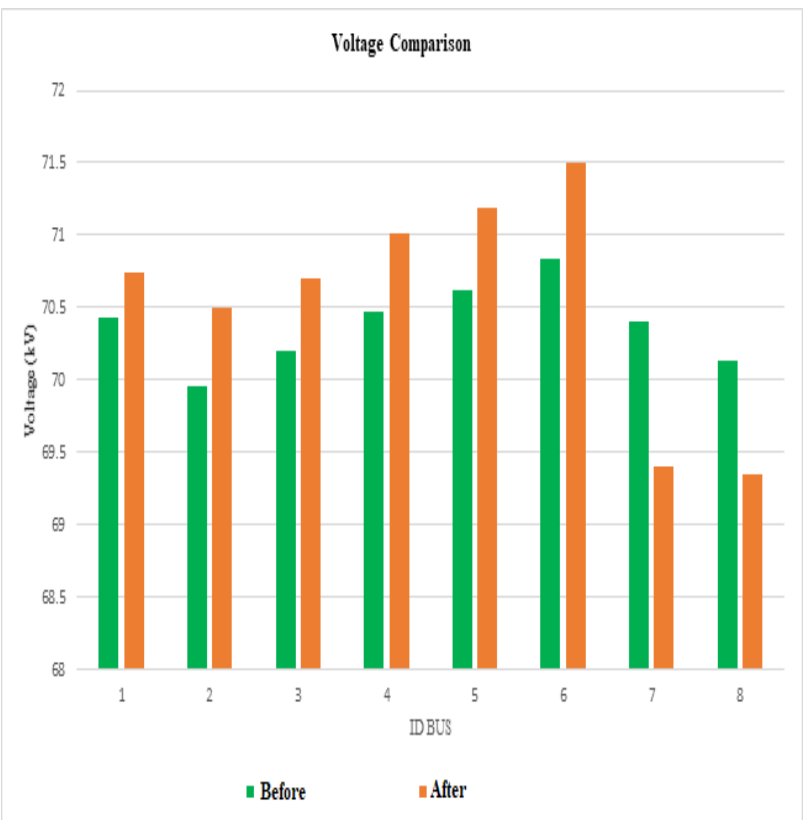

Fig. 2. 70kV voltage comparison graph from Power flow analysis and data from [3]
TABLE VII

Results Of Voltage Simulation Of 150KV INTERCONNECTION SYSTEM IN SULSELRABAR

\begin{tabular}{|c|c|c|c|c|}
\hline \multirow{2}{*}{ ID BUS } & \multicolumn{2}{|c|}{ Before } & \multicolumn{2}{|c|}{ After } \\
\hline & $\mathrm{kV}$ & $\%$ & $\mathrm{kV}$ & $\%$ \\
\hline BU28 & 149,895 & 99,93 & 149,475 & 99,65 \\
\hline BUS1 & 150 & 100 & 150 & 100,00 \\
\hline BUS2 & 149,511 & 99,67 & 150,721 & 100,48 \\
\hline BUS3 & 149,086 & 99,39 & 151,33 & 100,89 \\
\hline BUS4 & 150,38 & 100,25 & 149,729 & 99,82 \\
\hline BUS5 & 150,572 & 100,38 & 151,015 & 100,68 \\
\hline BUS6 & 150,831 & 100,55 & 151,256 & 100,84 \\
\hline BUS7 & 149,536 & 99,69 & 151,368 & 100,91 \\
\hline BUS8 & 147,783 & 98,52 & 150,894 & 100,60 \\
\hline BUS9 & 147,071 & 98,05 & 149,154 & 99,44 \\
\hline BUS10 & 147,537 & 98,36 & 149,165 & 99,44 \\
\hline BUS11 & 1477,51 & 98,35 & 148,775 & 99,18 \\
\hline BUS12 & 147,23 & 98,15 & 148,805 & 99,20 \\
\hline BUS13 & 147,802 & 98,53 & 149,679 & 99,79 \\
\hline BUS14 & 147,501 & 98,33 & 149,404 & 99,60 \\
\hline BUS15 & 148,379 & 98,92 & 151,836 & 101,22 \\
\hline BUS16 & 150,425 & 100,28 & 151,189 & 100,79 \\
\hline BUS17 & 149,339 & 99,56 & 149,041 & 99,36 \\
\hline BUS18 & 148,745 & 99,16 & 148,091 & 98,73 \\
\hline BUS19 & 148,809 & 99,21 & 152,199 & 101,47 \\
\hline BUS20 & 151,512 & 101,01 & 152,917 & 101,94 \\
\hline BUS21 & 151,064 & 100,71 & 152,022 & 101,35 \\
\hline BUS22 & 152,315 & 101,54 & 154,696 & 103,13 \\
\hline BUS23 & 151,244 & 100,83 & 154,735 & 103,16 \\
\hline BUS24 & 151,217 & 100,81 & 156,031 & 104,02 \\
\hline BUS25 & 148,83 & 99,22 & 154,396 & 102,93 \\
\hline BUS26 & - & - & 153,831 & 103,37 \\
\hline BUS27 & - & - & 154,103 & 102,74 \\
\hline BUS28 & - & - & 150,27 & 100,18 \\
\hline BUS29 & - & - & 149,643 & 99,76 \\
\hline BUSBNTAENG & - & - & 155,048 & 103,37 \\
\hline BusBOLANGI & - & - & 149,014 & 99,34 \\
\hline BUS26 & 70,424 & 100,61 & 70,744 & 101,06 \\
\hline BUS27 & 69,951 & 99,93 & 70,493 & 100,70 \\
\hline BUS29 & 70,2 & 100,29 & 70,702 & 101,00 \\
\hline BUS30 & 70,473 & 100,68 & 71,012 & 101,45 \\
\hline Bus31 & 70,625 & 100,89 & 71,189 & 101,70 \\
\hline BUS32 & 70,84 & 101,1 & 71,493 & 102,13 \\
\hline Bus33 & 70,4 & 100,57 & 69,396 & 99,14 \\
\hline BUS34 & 70,139 & 100,2 & 69,351 & 99,07 \\
\hline Bus35 & 29,43 & 98,1 & 29,411 & 98,04 \\
\hline
\end{tabular}

While the buses after the entry of the WPP experiencing Critical Voltage Conditions are found in two distribution buses, including:

1. Bosowa $11 \mathrm{kV}$ distribution bus of $9.7475 \mathrm{kV}$ or $88.61 \%$ experiencing Under Voltage Condition.

2. Pangkep $20 \mathrm{kV}$ distribution bus with 18,429 $\mathrm{kV}$ or $92.14 \%$ experiencing Under Voltage Condition. It is described in Table 8. 
TABLE VIII

Results Of Simulation Of The 150Kv INTERCONNECTION System Voltage In Sulselrabar After The Inclusion OF Wind Power With CRitical Voltage Condition

\begin{tabular}{cccc}
\hline \hline \multirow{2}{*}{ ID BUS } & \multicolumn{2}{c}{ VOLTAGE } & \multirow{2}{*}{ CONDITION } \\
\cline { 2 - 3 } & $\mathrm{kV}$ & $\%$ & \\
\hline BD_BOSOWA & 9,7475 & 88,6136 & Under Voltage \\
BD_PNGKEP & 18,4295 & 92,1475 & Under Voltage \\
\hline \hline
\end{tabular}

Marginal Voltage Condition events can be seen in the Table 9. Where for this condition, the voltage is still within the Standard Voltage, namely $+5 \%$ and $5 \%$ so that it is still allowed to operate. The following is the result of the calculation of the voltage for each bus that experiences Under Voltage or Over Voltage Conditions.

TABLE IX

Results Of Simulation Of THE 150Kv InTERCONNECTION System Voltage In Sulselrabar AfTER THE INCLUSION OF Wind POWER Which EXPERIENCES MARginal Voltage CONDITION

\begin{tabular}{lccc}
\hline \hline \multirow{2}{*}{ ID BUS } & \multicolumn{2}{c}{ VOLTAGE } & \multirow{2}{*}{ CONDITION } \\
\cline { 2 - 3 } & $\mathrm{kV}$ & $\%$ & \\
\hline BD1_PLPO & 19,5978 & 97,9889 & Under Voltage \\
BD1_PNGAYA & 20,5334 & 102,667 & Over Voltage \\
BD1_PNKNG & 19,506 & 97,53 & Under Voltage \\
BD1_TLAMA & 19,4464 & 97,2321 & Under Voltage \\
BD1_TLLASA & 19,5401 & 97,7006 & Under Voltage \\
BD2_PNKNG & 19,4102 & 97,0508 & Under Voltage \\
BD3_PNKNG & 19,4937 & 97,4685 & Under Voltage \\
BD_9 & 20,7842 & 103,921 & Over Voltage \\
BD_10 & 20,8039 & 104,019 & Over Voltage \\
BD_BLKMBA & 20,613 & 103,065 & Over Voltage \\
BD_BNTAENG & 20,5146 & 102,573 & Over Voltage \\
BD_BOLANGI & 19,4795 & 97,3976 & Over Voltage \\
BD_BRLOE & 20,5373 & 102,687 & Over Voltage \\
BD_BRWJA & 19,0058 & 95,0288 & Under Voltage \\
BD_MROS & 19,4486 & 97,2432 & Under Voltage \\
BD_SDRP & 19,2944 & 96,4719 & Under Voltage \\
BD_SNGKNG & 19,4049 & 97,0242 & Under Voltage \\
BD_SPPENG & 20,4878 & 102,439 & Over Voltage \\
BD_TBNGA & 19,5604 & 97,8022 & Under Voltage \\
BD_TELLO & 19,2802 & 96,4011 & Under Voltage \\
BD_TELLO2 & 19,3837 & 96,9186 & Under Voltage \\
BD_TONASA & 19,3177 & 96,5885 & Under Voltage \\
\hline \hline
\end{tabular}

\section{Conclusion}

The conclusion obtained from the results of Power Flow Analysis Power Flow Analysis Due to the Entry of Renewable Energy Plants in the Sulselrabar System Using ETAP 16, is:

1. The Newton-Raphson method used for power flow simulation in this study shows efficiency in terms of computational processing speed in ETAP 16.00 Software.

2. The total active power $(\mathrm{P})$ contained in the channel under normal conditions is 1730.87
MW, where the active power is the largest, which is $171 \mathrm{MW}$ from BUS15_TLASA to BUS13_SGMNSA.

3. The largest reactive power is $25.68 \mathrm{Mvar}$ from BUS8_PANKEP to BUS8_BOSOWA.

4. The highest Power Factor efficiency reached $100 \%$ occurred from BUS21_SENGKANG to BUS16_SIDRAP, while the lowest efficiency occurred on the WPP Sidrap Bus channel to BUS16_SIDRAP which was $19.72 \%$.

\section{Acknowledgements}

The authors would like to thank Ministry of Education, Culture, Research and Technology, director general of higher education, Director of Resources of Education, Culture, Research and Technology and Center for Research and Community Service State Polytechnic of Ujung Pandang for supporting the Research

\section{References}

S. D. Auliyani, A. Zulhajji, and A. Imran, " Rugirugi daya pada jaringan transmisi sistem interkoneksi Sulselrabar menggunakan program DIgSILENT," Diploma Thesis, Universitas Negeri Makassar, Makassar, 2020.

[2] M. F. S. Muhiddin, "Analisis aliran daya pada sistem jaringan transmisi Sulselbar dengan masuknya transmisi baru dari G.I Punnagaya ke G.I daya baru $275 \mathrm{KV}$," Universitas Hasanuddin, Makassar, 2019.

[3] M. R. Djalal, M. A. Haikal, T. M. P. N. U. Pandang, and T. E. I. P. Aceh, "Penyelesaian Aliran Daya 37 Bus Dengan Metode Newton Raphson (Studi Kasus Sistem Interkoneksi 150 kV Sulawesi Selatan)," 2014.

[4] M. R. Djalal, A. Imran, and I. Robandi, "Optimal placement and tuning power system stabilizer using participation factor and imperialist competitive algorithm in $150 \mathrm{kV}$ South of Sulawesi system," in Intelligent Technology and Its Applications (ISITIA), 2015 International Seminar on, 2015, pp. 147-152: IEEE.

[5] A. G. Nigara and Y. Primadiyono, "Analisis aliran daya sistem tenaga listrik pada bagian texturizing di PT Asia Pasific Fibers Tbk Kendal menggunakan Software ETAP Power Station 4.0," 2015.

[6] E. H. Harun, "Analisis tegangan setiap bus pada sistem tenaga listrik Gorontalo melalui simulasi aliran daya," JURNAL SAINSTEK UNIVERSITAS NEGERI GORONTALO, vol. 6, no. 6, 2012.

[7] J. Leda and S. Patabang, "Studi Aliran Daya Pada Sistem Kelistrikan Sulawesi Selatan". 2018. 
[8] P. Perinov, U. Situmeang, and M. Monice, "Analisis kontingensi sistem tenaga listrik riau menggunakan metode aliran daya newton raphson," Prosiding Seminar Nasional Pakar, no. Prosiding Seminar Nasional Pakar 2019 Buku I, pp. 1.23.1-1.23.8, 2019.

[9] B. T. Aribowo, S. Setiawidayat, and M. Muksim, "Simulasi dan analisis load flow sistem interkoneksi kalimantan timur menggunakan software ETAP 12.6," presented at the Conference on Innovation and Application of Science and Technology (CIASTECH), Malang, 2018.

[10] M. R. Djalal, H. Setiadi, D. Lastomo, and M. Y. Yunus, "Modal Analysis and Stability Enhancement of $150 \mathrm{kV}$ Sulselrabar Electrical System using PSS and RFB based on Cuckoo Search Algorithm," International Journal on Electrical Engineering and Informatics, vol. 9, no. 4, pp. 800-812, 2017.

\section{Authors' Information}

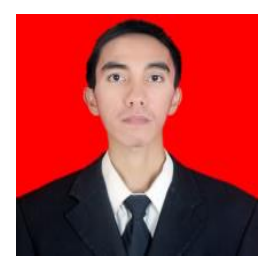

Muhammad Ruswandi Djalal was born in Makassar-Indonesia on march 11, 1990. He received bachelor degree from State Polytechnic of Ujung Pandang (Makassar, Indonesia), majors in Energy Generation engineering in 2012. Then, master degree from Sepuluh Nopember Institute of Technology, (ITS Surabaya, Indonesia), majors in Power System Engineering in 2015. His research about, Power System Operation and Control, Renewable Energy and Artificial Intelligent. Now, $\mathrm{He}$ is lecturer at State Polytechnic of Ujung Pandang (PNUP).

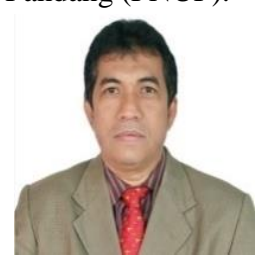

Makmur Saini received his B. Eng. in Electrical Engineering in 1987 from Hasanuddin University, Indonesia, M.Eng Electrical Power in 1993 from Institut Teknologi Bandung, Indonesia and Ph.D. degree from the University of Technology Malaysia, in 2016 also in electric power system. Currently, he is an Professor at Engineering Department of Mechanical Engineering, Politeknik Negeri Ujung Pandang, Indonesia. His research interests include Power System Protection, power system stability, Transmission and Distribution, High Voltage and renewable energy application. Dr. Makmur Saini also acts as general secretary of the Indonesian Electric Power Expert Association for South Sulawesi chapter since 2016 and active as an assessor in Power Distribution System.

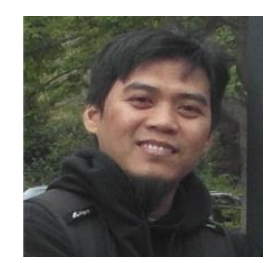

A. M. Shiddiq Yunus received his bachelor degree in Electrical Engineering in 2000 at Hasanuddin University, Indonesia with Honor. His master and doctoral degree were received at Queensland University of Technology (QUT) Australia and Curtin University Australia in 2006 and 2012 respectively, both in Electrical Engineering. Currently, he is working for Energy Conversion Study Program, Mechanical Engineering Department, State Polytechnic of Ujung Pandang, Indonesia. He has become a regular reviewer for IEEE Transaction Journal, IET Journal and Australian Journal of Electrical and Electronics Engineering since 2012. He was a recipient of QUT prize for International Student with The Highest GPA Achievement in his/her Bachelor Degree in 2006. He was a recipient of Best Presenter Award in AUPEC Conference 2012 and Best Paper Award in ICBIIEM Conference at Pahang Malaysia 2018. He was also a recipient of Nuffic Fellowship Program (NFP) in 2015. Dr. A. M. Shiddiq Yunus is a member of Indonesian Electric Power Expert Association since 2007 and acted as an assessor in Power Distribution System. 